\title{
Massive cerebral venous thrombosis related to oligosymptomatic COVID-19 infection: a case report
}

Simone Beretta ( $\nabla$ simone.beretta@unimib.it )

University of Milano Bicocca https://orcid.org/0000-0002-9417-2748

\section{Fulvio Da Re}

University of Milano Bicocca

Valentina Francioni

University of Milano Bicocca

Paolo Remida

San Gerardo Hospital ASST Monza

\section{Benedetta Storti}

University of Milano Bicocca

Lorenzo Fumagalli

San Gerardo Hospital ASST Monza

Maria Luisa Piatti

San Gerardo Hospital ASST Monza

\section{Patrizia Santoro}

San Gerardo Hospital ASST Monza

\section{Diletta Cereda}

San Gerardo Hospital ASST Monza

\section{Claudia Cutellè}

University of Milano Bicocca

\section{Fiammetta Pirro}

University of Milano Bicocca

\section{Danilo Antonio Montisano}

University of Milano Bicocca

Francesca Beretta

University of Milano Bicocca

Francesco Pasini

University of Milano Bicocca

Annalisa Cavallero

San Gerardo Hospital ASST Monza

Ildebrando Appollonio

University of Milano Bicocca

Carlo Ferrarese 


\section{Case Report}

Keywords: COVID-19, cerebral venous thrombosis, anticoagulation, thromboinflammation

Posted Date: July 29th, 2020

DOI: https://doi.org/10.21203/rs.3.rs-49755/v1

License: (c) (1) This work is licensed under a Creative Commons Attribution 4.0 International License. Read Full License 


\section{Abstract}

Background: The development of thrombotic coagulopathy is frequent in COVID-19 patients, but the timing after infection, cerebral venous system involvement, treatment and outcome are uncertain.

Case Presentation: We report a case of massive cerebral venous thrombosis occurring in the late phase of COVID-19 infection. Mild respiratory symptoms, without fever, started three weeks before headache and acute neurological deficits. She had no dyspnea, although she was hypoxic and with typical COVID19 associated interstitial pneumonia. Brain CT scan showed a left parietal hypodense lesion with associated sulcal subarachnoid haemorrhage. CT angiography showed a massive cerebral vein thrombosis. An asymptomatic concomitant right internal iliac vein thrombosis was found. Both cerebral venous thrombosis and deep venous thrombosis were effectively treated with unfractionated heparin started on the day of admission, then shifted to low molecular weight heparin, with a favorable clinical course. Nasopharyngel swab, repeated twice, tested negative for SARS-CoV-2. Serological tests confirmed SARS-CoV-2 infection.

Conclusions: Our case supports active surveillance and prevention of thrombotic complications associated with COVID-19, which may affect both peripheral and cerebral venous system. Early initiation of unfractionated heparin may lead to good neurological outcome.

\section{Background}

At the time of this writing, health care systems are facing worldwide the pandemic of the coronavirus SARS-CoV-2 and its associated disease, named COVID-19 [1]. Although COVID-19 mostly affects the respiratory system, ranging from mild flu-like symptoms to severe pneumonia, the development of thrombotic coagulopathy is frequent in COVID-19 patients and highly associated with poor prognosis [23]. Here, we report the case of a massive cerebral venous thrombosis occurring in the late phase of COVID-19 infection.

\section{Case Presentation}

A 62 years old female patient was referred to the emergency room for acute onset of confusion, dysarthria, and right limbs weakness. In the previous three weeks she had dry cough, fatigue and loss of appetite. In the last few days, she complained of a headache. Fever was not reported. Her medical history included arterial hypertension.

Neurological examination showed a moderately agitated patient with global aphasia, right-sided neglect and a severe hypotonic right hemiparesis (MRC power scale 1/5 at right upper limb and 0/5 at right lower limb). Arterial blood gases showed hypoxia (paO2 $59 \mathrm{mmHg}$ breathing room air) despite she had no dyspnea. D-dimer levels were high $(2768 \mathrm{ng} / \mathrm{mL})$ and reactive $C$ protein was moderately elevated $(19.45$ $\mathrm{mg} / \mathrm{dL}$ ). Brain CT showed a left parietal hypodense lesion and a sulcal subarachnoid haemorrhage over the left temporal lobe. CT cerebral venography showed a massive cerebral vein thrombosis (CVT) 
involving the right transverse sinus, the right jugular bulb, the superior sagittal sinus, the straight sinus, the vein of Galen, and both internal cerebral veins (Fig. 1A). Chest CT showed multiple bilateral ground-

glass opacities and consolidations typical of COVID-19 pneumonia. Nasopharyngel swab, repeated twice, tested negative for SARS-CoV-2. Infectious disease specialist was consulted and diagnosed COVID-19 on the base of typical chest CT findings, blood tests and recent history of cough and malaise.

A diagnosis of massive CVT associated with COVID-19 was made and she was admitted to the Acute Stroke Unit. On the day of admission, anticoagulation therapy was started with full-dose intravenous unfractionated heparin (UFH), then shifted to subcutaneous low molecular weight heparin (LMWH) after 5 days. Antibiotics (ceftriaxone and azithromycin), anti-viral therapy (lopinavir + ritonavir) and hydroxychloroquine were also started on the same day. Low dose supplemental oxygen (24-28\%) was administered, with no need of further respiratory support. Abdominal and pelvic CT showed no malignancy but revealed a right internal iliac vein thrombosis. Screening tests for a thrombophilic state were not performed, since interference with heparin prevented their interpretation. Both CVT and peripheral vein thrombosis responded well to anticoagulation therapy. Clinical course was favourable with gradual improvement in language, cognition and right motor deficit. Follow-up brain CT and CT angiography showed reduction of venous infarct volume, resolution of subarachnoid hemorrhage and partial venous recanalization (Fig. 1B). She was discharged 3 weeks later in a rehabilitation center with the advice to continue anticoagulation therapy. Serological test was performed on day 38 after admission and confirmed SARS-Cov-2 infection.

\section{Discussion And Conclusions}

We report a possible correlation between COVID-19 infection and CVT, a condition that has been linked to ear and nose bacterial infections in the past and, rarely, to viral brain infections [4]. Our case suggests that hypercoagulability and thromboinflammation associated with COVID-19 [5] may cause CVT in the late phase of infection, a few weeks after the onset of mild respiratory symptoms. In the last month, a few cases of COVID-19 associated CVT were reported, mostly with fatal or unreported outcome [6-10]. In our case, CVT occurred simultaneously with an asymptomatic internal iliac vein thrombosis and both venous thromboses were effectively treated with intravenous UFH followed by subcutaneous LMWH.

\section{Abbreviations}

SARS-CoV-2: severe acute respiratory coronavirus 2; COVID-19; coronavirus disease 19; MRC: medical research council; CT: computerized tomography; CVT: cerebral venous thrombosis; LMVH: low molecular weight heparin.

\section{Declarations}

Acknowledgement 
The authors thank medical and nursing staff of the Acute Stroke Unit and Covid Intermediate Care Unit, San Gerardo Hospital ASST Monza, for their support and collaboration.

\section{Authors' contributors}

SB and FDR had the idea for the paper. SB prepared the first draft with FDR and VF. PR performed imaging acquisition and interpretation, prepared the figure and critically reviewed the manuscript for intellectual content. SB, FDR, VF, BS, LF, MLP, PS, DC, CC, FP, DM, FB, FP were involved in the clinical care of the patients and critically reviewed the manuscript for intellectual content. AC performed the virological and serological tests and critically reviewed the manuscript for intellectual content. IA and CF assisted with imaging and case interpretation and critically reviewed the manuscript for intellectual content.

\section{Funding}

The authors have not declared a specific grant for this research from any funding agency in the public, commercial or not-for-profit sectors.

\section{Availability of data and materials}

Upon request to the corresponding author.

\section{Consent for publication}

Written informed consent was obtained from the patient for the publication of this case report.

\section{Competing interests}

The authors declare that they have no competing interests.

\section{References}

1. Huang C, Wang Y, Li X, et al. (2020) Clinical features of patients infected with 2019 novel coronavirus in Wuhan, China. Lancet 395:497-506.

2. Tang N, Li D, Wang X, et al. (2020) Abnormal coagulation parameters are associated with poor prognosis in patients with novel coronavirus pneumonia. J Thromb Haemost 18:844-847.

3. Zhai Z, Li C, Chen Y, et al. (2020) Prevention and Treatment of Venous Thromboembolism Associated with Coronavirus Disease 2019 Infection: A Consensus Statement before Guidelines. Thromb Haemost. doi:10.1055/s-0040-1710019.

4. Vedani M, Toubas O, Hentzien M, et al. (2020) Cerebral venous thrombosis: a rare complication of herpes simplex encephalitis. J Neurovirol 26:114-117.

5. Connors JM, Levy JH (2020) Thromboinflammation and the hypercoagulability of COVID-19. J Thromb Haemost. doi:10.1111/jth.14849. 
6. Chougar L, Mathon B, Weiss N, Degos V, Shor N. (2020) Atypical Deep Cerebral Vein Thrombosis with Hemorrhagic Venous Infarction in a Patient Positive for COVID-19. Am J Neuroradiol. doi:10.3174/ajnr.A6642

7. Cavalcanti DD, Raz E, Shapiro M, et al. (2020) Cerebral Venous Thrombosis Associated with COVID19. Am J Neuroradiol. 2020. doi:10.3174/ajnr.A6644

8. Malentacchi M, Gned D, Angelino V, et al. (2020) Concomitant brain arterial and venous thrombosis in a COVID-19 patient. Eur J Neurol. doi:10.1111/ene.14380

9. Poillon G, Obadia M, Perrin M, Savatovsky J, Lecler A (2020) Cerebral venous thrombosis associated with COVID-19 infection: Causality or coincidence? J Neuroradiol. doi:10.1016/j.neurad.2020.05.003

10. Garaci F, Di Giuliano F, Picchi E, Da Ros V, Floris R (2020) Venous cerebral thrombosis in COVID-19 patient. J Neurol Sci 414:116871.

\section{Figures}
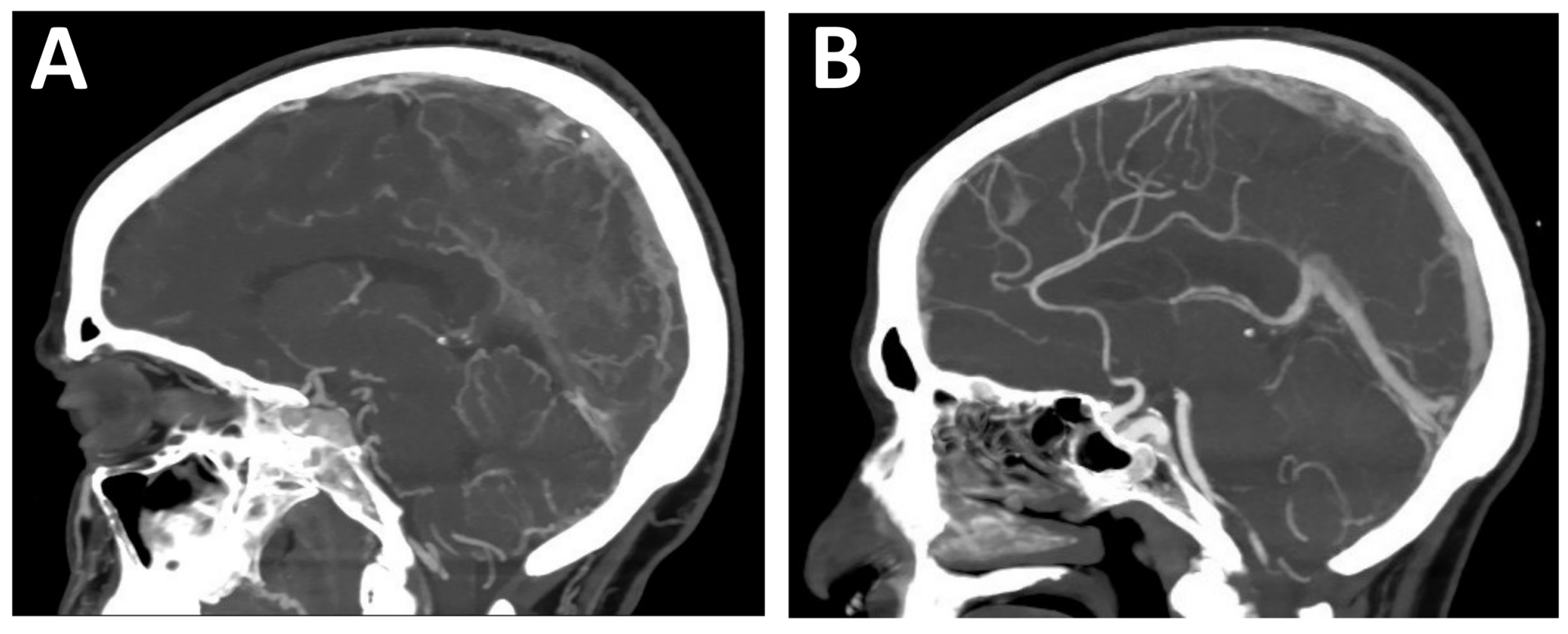

Figure 1

Representative CT cerebral venography images of cerebral venous thrombosis associated with COVID-19 infection. A. At admission. B. After 3 weeks of anticoagulation therapy. 